\title{
Three-dimensional visualization improves the endoscopic diagnosis of superficial gastric neoplasia
}

\author{
Kazutoshi Higuchi ${ }^{*}$, Mitsuru Kaise ${ }^{1}$, Hiroto Noda ${ }^{1}$, Kumiko Kirita' ${ }^{1}$, Eriko Koizumi ${ }^{1}$, Takamitsu Umeda ${ }^{1}$, \\ Teppei Akimoto ${ }^{1}$, Jun Omori ${ }^{1}$, Naohiko Akimoto ${ }^{1}$, Osamu Goto ${ }^{1}$, Atsushi Tatsuguchi ${ }^{1,2}$ and Katsuhiko Iwakiri ${ }^{1}$
}

\begin{abstract}
Background: Three-dimensional (3D) technology has been used in many fields, including flexible endoscopy. We evaluated the usefulness of 3D visualization for endoscopically diagnosing superficial gastric neoplasia.

Methods: Twelve participants ( 4 novices, 4 trainees and 4 experts) evaluated two-dimensional (2D) and 3D endoscopic still images of 28 gastric neoplasias, obtained before ESD with white-light imaging (WLI) and narrow-band imaging (NBI). Assessments of the delineation accuracy of tumor extent and tumor morphology under 2D and 3D visualization were based on the histopathological diagnosis of ESD specimens. Participants answered visual analog scale (VAS) questionnaires (0-10, worst to best) concerning the (a) ease of recognition of lesion morphology, (b) lesion extent and (c) comprehensive endoscopic cognition under 2D and 3D visualization. The endpoints were the accuracy of tumor extent and morphology type and the degree of confidence in assessing (a)-(c).

Results: The delineation accuracy of lesion extent [mean (95\% confidence interval)] with WLI under 3D visualization [60.2\% (56.1-64.3\%)] was significantly higher than that under 2D visualization [52.3\% (48.2-56.4\%)] $(P<0.001)$. The accuracy with NBI under 3D visualization [70.3\% (66.8-73.7\%)] was also significantly higher than that under 2D visualization [64.2\% (60.7-67.4\%)] $(P<0.001)$. The accuracy of the morphology type with NBI under 3D visualization was significantly higher than that under $2 \mathrm{D}$ visualization $(P=0.004)$. The VAS for all aspects of endoscopic recognition under 3D visualization was significantly better than that under 2D visualization $(P<0.01)$.
\end{abstract}

Conclusions: Three-dimensional visualization can enhance the diagnostic quality for superficial gastric tumors.

Keywords: Three-dimensional endoscopy, Two-dimensional endoscopy, Early gastric cancer, Cancer extent

\section{Background}

Gastric cancer is the fifth-most commonly diagnosed cancer worldwide and the third-most common cause of cancer-related death [1]. Early detection of gastric cancer is key to reducing the rate of cancer death. Early gastric cancer (EGC) without lymph node metastasis can be

\footnotetext{
*Correspondence: k-higuchi@nms.ac.jp

${ }^{1}$ Department of Gastroenterology, Nippon Medical School, Graduate School of Medicine, 1-1-5, Sendagi, Bunkyo-ku, Tokyo 113-8603, Japan Full list of author information is available at the end of the article
}

cured by endoscopic submucosal dissection (ESD), and the quality of life after ESD is quite favorable [2,3].

Detecting gastric cancer in an early stage and precisely delineating the cancer margin are necessary for a high cure rate by ESD. Novel technologies and devices have improved the quality of the endoscopic diagnosis. Optical image-enhanced technologies, such as narrow-band imaging (NBI), blue laser imaging and linked color imaging have facilitated the diagnosis of superficial gastric neoplasia [4-7]. The technology of three-dimensional (3D) visualization is currently being used in many fields, 
including movies, video games and show attractions in amusement parks, and has also been introduced to some medical fields.

The technique of 3D rigid endoscopy is clinically used in surgical fields and has enabled a more accurate and speedy procedure than a two-dimensional (2D) approach $[8,9]$. Furthermore, this approach has been introduced in robotic surgery, enhancing the precision of minimally invasive surgery [10]. In addition, 3D flexible endoscopy has also been developed. In an ex vivo animal model of ESD, 3D visualization shortened the procedure time and decreased the adverse event rate compared to 2D, especially in trainees [11-13]. Recently, 3D flexible endoscopy was clinically applied in humans and reported to be feasible for ESD [14]. However, while several studies have recently addressed the efficiency of 3D flexible endoscopy in this procedure [15], very few clinical studies have evaluated the usefulness for making an endoscopic diagnosis.

The present study evaluated the usefulness of 3D visualization for determining the margin of superficial gastric neoplasia compared to $2 \mathrm{D}$ visualization in clinical practice.

\section{Materials and methods}

\section{Enrolled lesions and endoscopic images}

Twenty-nine superficial gastric neoplasias excised by ESD in Nippon Medical School Hospital from March 2018 to January 2019 were serially enrolled. Endoscopic still images of the enrolled lesions were obtained before ESD marking with 2D white-light imaging (WLI), 3D-WLI, 2D-NBI and 3D-NBI. The 2D and 3D images of each lesion were exactly same aside from being obtained via $2 \mathrm{D}$ or $3 \mathrm{D}$ visualization. This was possible because the 3D image set and corresponding 2D image set were both preserved when a still image was taken with the $3 \mathrm{D}$ endoscopy system. A set of representative 2D-WLI, 3D-WLI, 2D-NBI and 3D-NBI images from each lesion was extracted from these images and used in this study. After the endoscopic observation, all lesions were excised by ESD and pathologically evaluated, including an assessment of the tumor extent diagnosis. The pathological tumor margin was delineated on a color print of the representative $2 \mathrm{D}$ image of each lesion in advance.

\section{Examination protocol}

The primary endpoint of this study was the accuracy of the tumor extent diagnosis assessed by the concordance rate between the pathological tumor margin and the endoscopically assumed tumor margin under 2D or 3D visualization. Study participants drew an endoscopically assumed tumor margin on a color print of a 2D image of an enrolled lesion while viewing the endoscopic still image on the video monitor under $2 \mathrm{D}$ or $3 \mathrm{D}$ visualization
(Fig. 1a, b). The concordance between the pathological and drawn margins was then evaluated on preset lines of the color print that corresponded to the tissue section lines of 2-mm-wide strips of ESD specimens (Fig. 1c). If the pathological tumor margin and drawn margin matched on the preset line, then it was assessed as being correct. If they did not match, it was assessed as being incorrect (Fig. 1d). If the drawn line was outside the pathological margin and intersected with the next adjacent preset line, the number of these intersecting points was counted as evaluation points and, then these points were assessed as being incorrect (Fig. 2a). If the drawn line was inside the pathological margin and did not pass the preset line, the evaluation points that the drawn lines did not pass through were assessed as being incorrect (Fig. 2b). The concordance rate was calculated as the number of correct points divided by the number of total evaluation points on the lines. The concordance rate was used as an index of the accuracy of the lesion extent diagnosis.

Twelve study participants were blinded to the endoscopic findings and histopathological diagnoses of the enrolled superficial gastric neoplasias. These participants were 4 novices (young physicians with no endoscopic practice), 4 trainees (physicians with experience performing ESD in 30-300 cases) and 4 experts (endoscopists with experience performing ESD in over 300 cases and Board-certified Fellows of the Japan Gastroenterological Endoscopy Society). A crossover design for evaluating the 2D and 3D images was used to reduce bias.

The novices, trainees and experts were divided equally into two groups (A and B). Group A evaluated 2D images first, followed by $3 \mathrm{D}$ images, while group $\mathrm{B}$ evaluated $3 \mathrm{D}$ images first, followed by 2D images. To reduce the carryover effect between 2 and 3D observation, the first and the second observations were interrupted by an interval exceeding four weeks as a wash-out period (Fig. 3). After finishing the delineation of the tumor margin, participants described the morphology type of the lesions and responded to visual analog scale (VAS) questionnaires concerning endoscopic lesion recognition, which was evaluated based on three aspects: (a) ease of recognition of lesion morphology, (b) ease of recognition of lesion extent and (c) ease of recognition of comprehensive endoscopic cognition. The VAS ranged from 0 to 10 (worst to best). If the endoscopists found the endoscopic observation very difficult, the rating was 0 . If they found it very easy, the rating was 10 . If they found it neither difficult nor easy, the rating was 5 . The morphology type was evaluated according to the macroscopic type classification described in Japanese classification of gastric carcinoma [16]. In this study, we used the pathological morphology as the final morphology of tumors. The pathological typing of tumor morphology was done by pathologists, who 

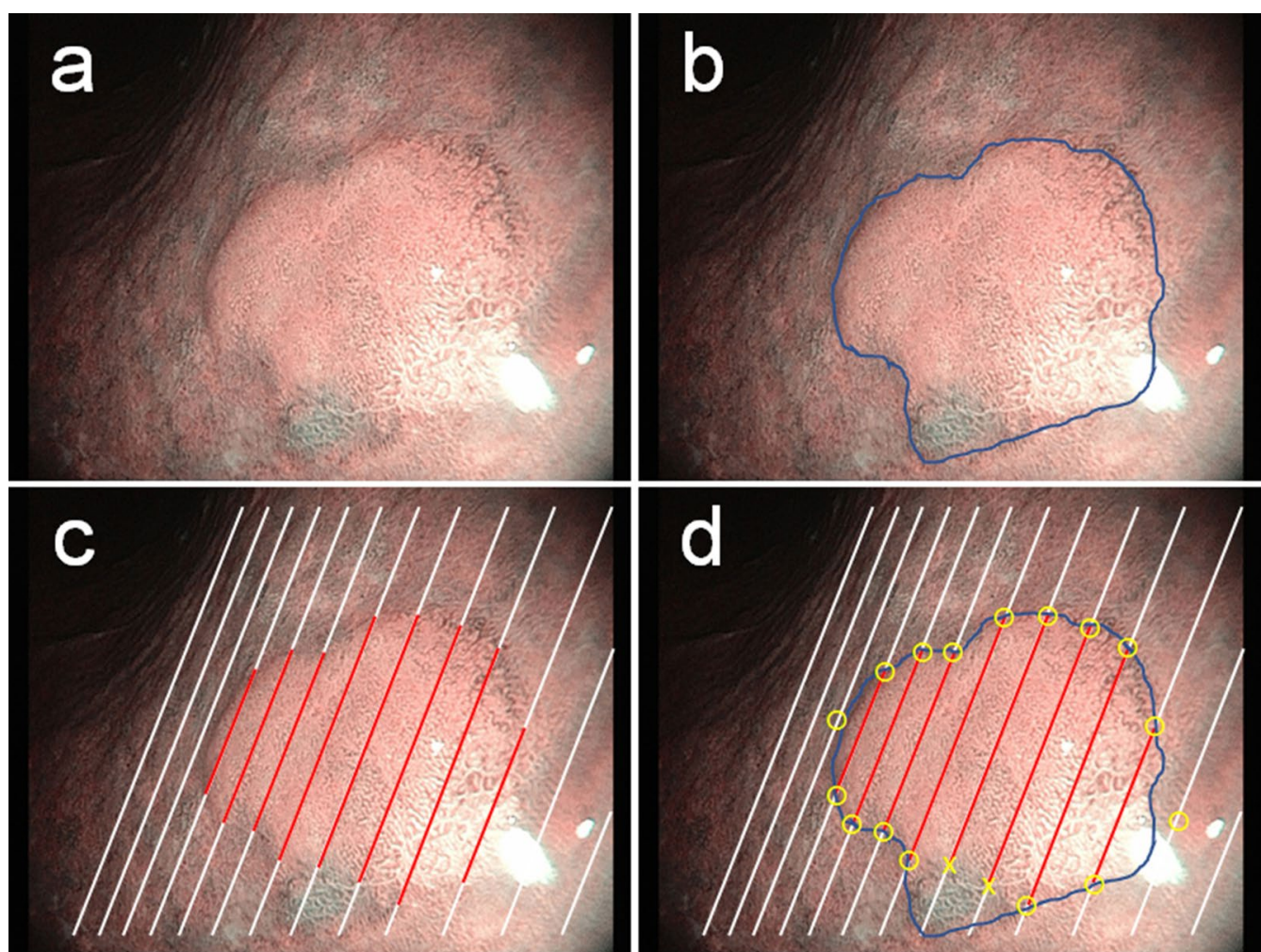

Fig. 1 Definition of the concordance rate of lesion extent. a Endoscopic image of a gastric neoplasia using NBI. A superficial protruded-type lesion (adenoma) is evident on the lesser curve of the lower gastric body. $\mathbf{b}$ Delineation of the tumor margin is performed. The blue line is the tumor extent line drawn by a participant. c According to the histopathological findings, the tumor extent (red lines) is reconstructed on an endoscopic image. The white lines are the cutting lines of a resected specimen. $\mathbf{d}$ The calculation of the concordance rate of lesion extent. Yellow circles and yellow cross marks show coincident and non-coincident evaluating points, respectively. In this case, 16 of the 18 evaluation points were coincident, indicating a coincident rate of lesion extent of $88.9 \%(16 / 18)$

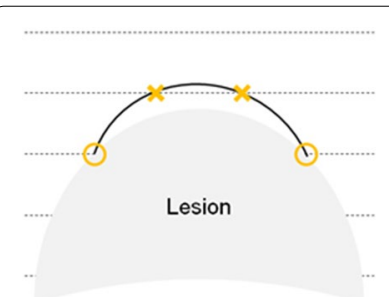

a

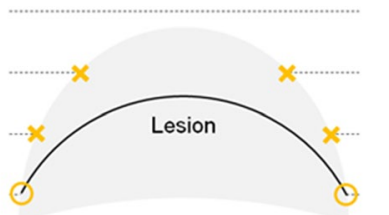

b
Fig. 2 Definition of the evaluation points for outside the lesion. a If the drawn line passes through the cutting lines outside the actual tumor margin, the intersections are evaluated as incorrect points. The black line shows the drawn line and dotted lines show the cutting lines. The yellow circles and yellow cross marks show the correct and incorrect evaluation points, respectively. $\mathbf{b}$ If the drawn line passes inside the tumor margin, the evaluation points that the drawn line do not pass through on the lesion border are evaluated as incorrect points macroscopically viewed the mucosal surface and also microscopically reviewed the lesion. Participants were given $30 \mathrm{~s}$ per lesion for the evaluation. During the time, they were able to observe the image of the lesion on the video monitor as many times as they wanted. After finishing the evaluation of one lesion, the next evaluation of another lesion was performed. The order of lesions to be displayed was randomly assigned. Participants evaluated WLI and NBI images of all enrolled lesions.

\section{Outcome measurement and sample size}

The primary endpoint of this study was the coincidence rate of the lesion extent. The accuracy of morphology type and ease of endoscopic lesion recognition were secondary endpoints. The sample size was not calculated because this was the first clinical pilot study, and no similar studies were available for reference. Around 30 lesions had been tentatively assumed as the target enrollment number along with the numbers enrolled in previous 3D endoscopy studies that involved comparisons with $2 \mathrm{D}$ endoscopy. 


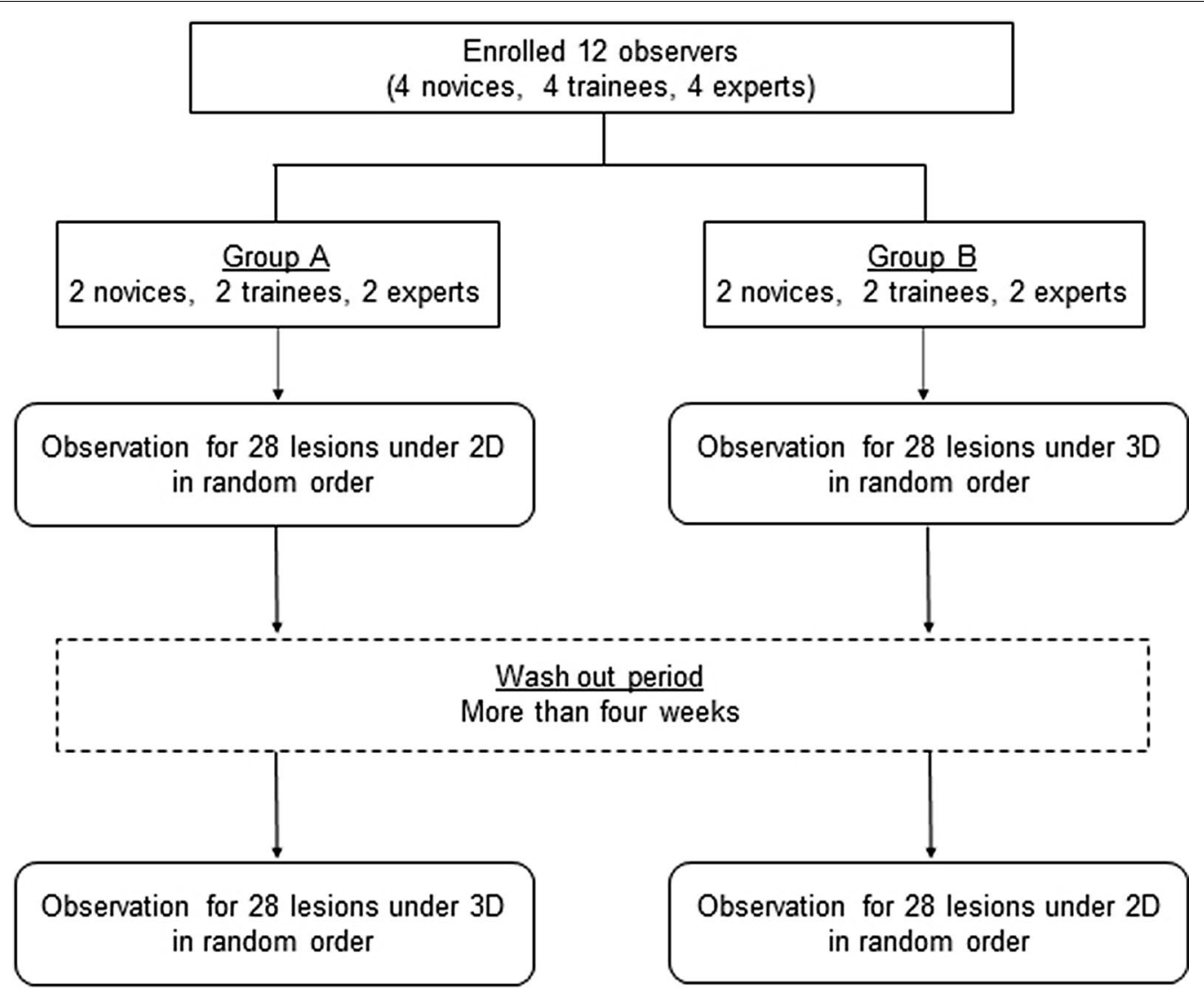

Fig. 3 Flow chart showing the enrollment of the participants and evaluation of endoscopic images

\section{D endoscopy system}

A 3D endoscope with stereoscopic optical system (GIF-Y0080; Olympus Medical Systems Corp., Tokyo, Japan) was used in this study (Fig. 4a). The electrical signal of the image obtained through each lens was transmitted to a video system center (EVIS EXERA III Video System Center, CV-190; Olympus Medical Systems Corp.) and synthesized in a 3D Visualization Unit (3DV-190; Olympus Medical Systems Corp.) (Fig. 4b). Endoscopists with 3D glasses were able to review the 3D images on a 3D monitor (LMD-2451MT; Sony, Tokyo, Japan) (Fig. 4c).

\section{Statistical analyses}

All variables were presented as the mean and standard deviation. Between-group comparisons were performed using Wilcoxon's signed-rank test for continuous variables and McNemar's test for categorical variables. $P$ values $<0.05$ were considered to be statistically significant. All statistical analyses were performed using the SPSS statistical software package (version 25; IBM Corp., New York, NY, USA).

\section{Results}

The en bloc resection rate by ESD of the 29 enrolled lesions was $100 \%$. One enrolled lesion was excluded because no neoplasia was found on the resected specimen by a histopathological evaluation. Therefore, the endoscopic images of 28 lesions were ultimately evaluated by the participants, equating to112 images of 2D-WLI, 3D-WLI, 2D-NBI and 3D-NBI. A pathological examination revealed that the 28 excised lesions consisted of 26 EGCs (21 well- and moderately differentiated adenocarcinomas, 4 Signet-ring cell carcinomas and 1 poorly differentiated adenocarcinoma) and 2 gastric adenomas. The mean lesion size was $14.8 \pm 7.6 \mathrm{~mm}$. The morphology types were superficial protruded type in 4, flat type in 3 and superficial depressed type in 21 (Table 1).

The concordance rate of the lesion extent with WLI under 3D visualization [60.2\%, 95\% confidence interval (CI) 56.1-64.3\%] was significantly higher than that under 2D visualization (52.3\%, 95\% CI 48.2-56.4\%) $(P<0.001)$, and that with NBI under 3D visualization $(70.3 \%$, 95\% CI 66.8-73.7\%) was also significantly higher than that under $2 \mathrm{D}$ visualization $(64.2 \%, 95 \% \mathrm{CI}$ 


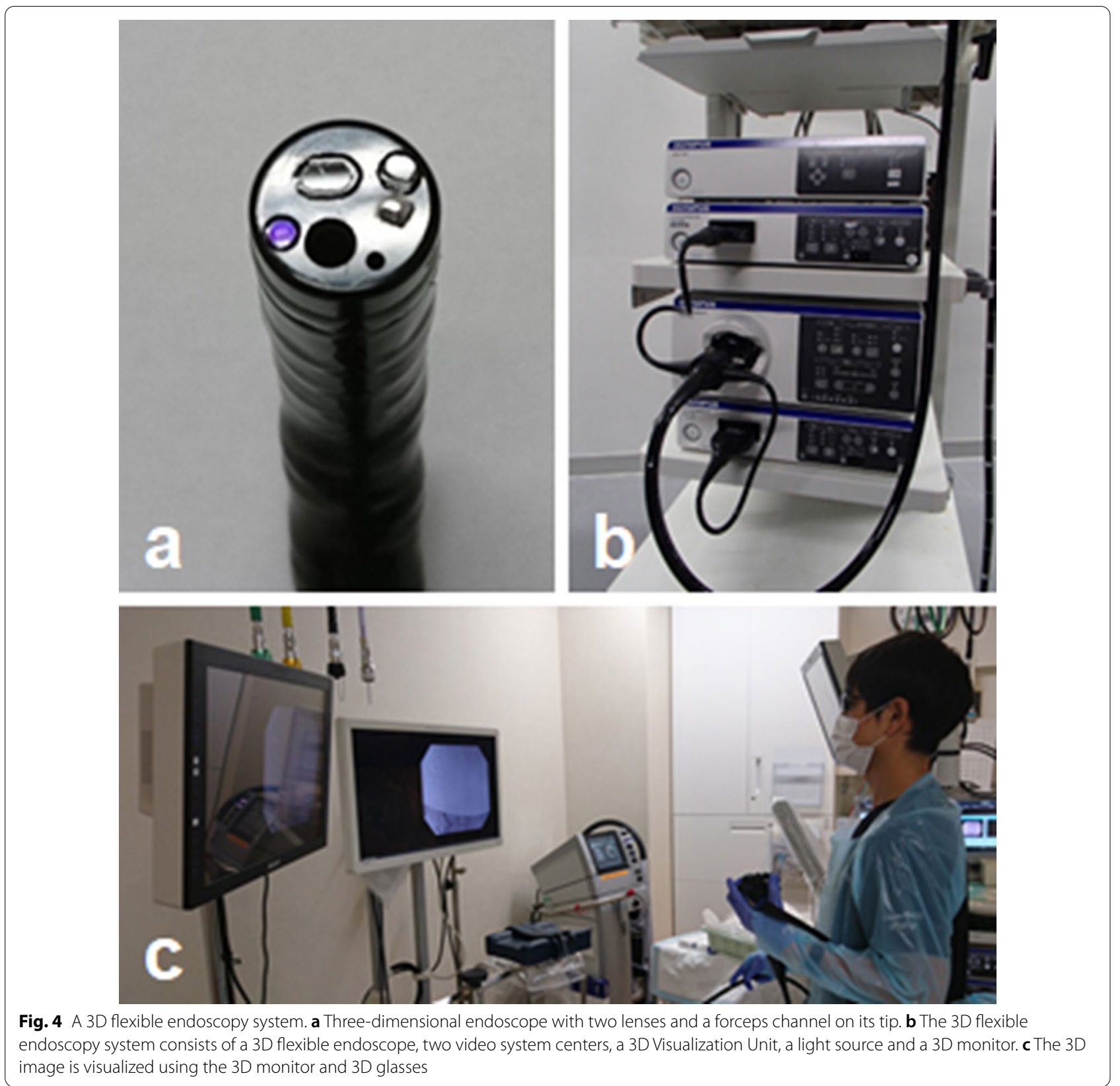

60.7-67.4\%) $(P<0.001)$. There were no significant differences between group A and Group B on 2D-WLI, 2D-NBI, 3D-WLI and 3D-NBI, respectively (Additional file 1: Supplementary Table 1).

The subgroup analysis with the skill level of endoscopy is shown in Table 2. In the novices, the tumor extent diagnosis under 3D-WLI was significantly superior to that under 2D-WLI, but the difference was not significant under NBI. In the experts, the superiority of $3 \mathrm{D}$ over $2 \mathrm{D}$ visualization was significant under NBI but small under WLI. In the trainees, the superiority of 3D over 2D visualization was most significant with about $10 \%$ addition under WLI and NBI observation.

The subgroup analysis with the morphology type is shown in Table 3. In depressed type, the tumor extent diagnosis under 3D visualization with both WLI and NBI was more accurate than that under $2 \mathrm{D}$ visualization (WLI: $53.2 \%$ vs. $43.4 \%$, NBI: $68.7 \%$ vs. $61.5 \%$, respectively $P<0.001)$. In flat type, the superiority of $3 \mathrm{D}$ over $2 \mathrm{D}$ visualization was not found under WLI or NBI because the accuracy rates were poor under $3 \mathrm{D}$ visualization. In protruded type, the superiority of 3D over $2 \mathrm{D}$ visualization 
Table 1 The characteristics of the lesions

\begin{tabular}{lc}
\hline En bloc resection rate, \% (n) & $100 \%(28 / 28)$ \\
Lesion size, mm & $14.8(7.6)$ \\
Specimen size, mm & $44.0(12.8)$ \\
Location & \\
Upper third, $\mathrm{n}(\%)$ & $3(10.7 \%)$ \\
Middle third, $\mathrm{n}(\%)$ & $13(46.4 \%)$ \\
Lower third, $\mathrm{n}(\%)$ & $12(42.9 \%)$ \\
Histological type & \\
Well- and moderately differentiated adenocarcinoma, & $21(75.0 \%)$ \\
$\quad \mathrm{n}(\%)$ & \\
Signet-ring cell carcinoma, $\mathrm{n}(\%)$ & $4(14.3 \%)$ \\
Poorly differentiated adenocarcinoma, $\mathrm{n}(\%)$ & $1(3.6 \%)$ \\
Adenoma, $\mathrm{n}$ (\%) & $2(7.1 \%)$ \\
Morphology type & \\
Superficial protruded type, $\mathrm{n}(\%)$ & $4(14.3 \%)$ \\
Flat type, $\mathrm{n}$ (\%) & $3(10.7 \%)$ \\
Superficial depressed type, $\mathrm{n}(\%)$ & $21(75.0 \%)$ \\
\hline
\end{tabular}

Data are presented as the mean (standard deviation)

was found under NBI but not under WLI because the accuracy rates under 2D-WLI reached $95.8 \%$.

The accuracy of the morphology type diagnosis with NBI under 3D visualization (76.0\%, 95\% CI 71.0-80.0\%) was significantly higher than that under $2 \mathrm{D}$ visualization (69.0\%, 95\% CI 63.0-74.0\%) $(P=0.004)$. In contrast, there was no significant difference between $2 \mathrm{D}(66.0 \%, 95 \% \mathrm{CI}$
61.0-72.0\%) and 3D (71.0\%, 95\% CI 66.0-76.0\%) visualization under WLI $(P=0.104)$ (Table 4$)$.

In the VAS, the (a) ease of recognition of lesion morphology, (b) ease of recognition tumor extent and (c) ease of recognition of total endoscopic cognition were higher under 3D visualization than under $2 \mathrm{D}$ visualization with both WLI and NBI $(P<0.001$, respectively) (Table 5$)$. The comparison by skill level is shown in Additional file 1: Supplementary Table 2.

\section{Discussion}

The present study revealed that the endoscopic recognition of the lateral margin of superficial gastric neoplasia under 3D visualization was more accurate and easier than that under 2D visualization, particularly in superficial depressed type. In addition, morphology recognition in NBI was easier under 3D than 2D visualization. To our knowledge, this is the first study to show the usefulness of $3 \mathrm{D}$ visualization for the endoscopic diagnosis of gastric neoplasia in clinical practice.

ESD is an excellent minimally invasive treatment for EGC without lymph node metastasis, and the range of indications has expanded with the development of technologies and devices associated with this technique. However, an inaccurate recognition of the tumor margin may result in incomplete resection with a positive margin for tumor cells, which can result in local recurrence. The precise recognition of differences in superficial morphology under $3 \mathrm{D}$ visualization enables the more accurate

Table 2 The coincidence rate of lesion extent

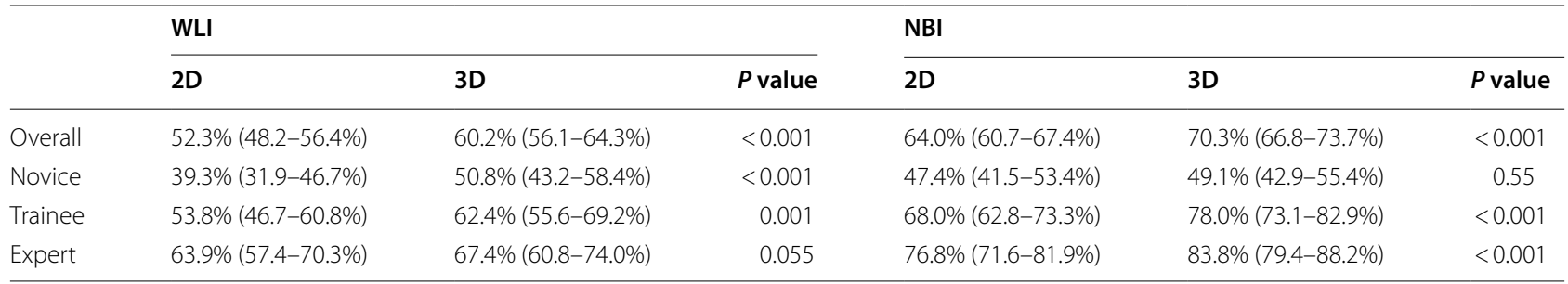

Data are presented as the mean (95\% confidence interval)

$W L /$ white-light imaging, $N B /$ narrow-band imaging, 2D 2-dimensional, 3D 3-dimensional

Table 3 The comparison by the morphology of the accuracy in assessing lesion extent

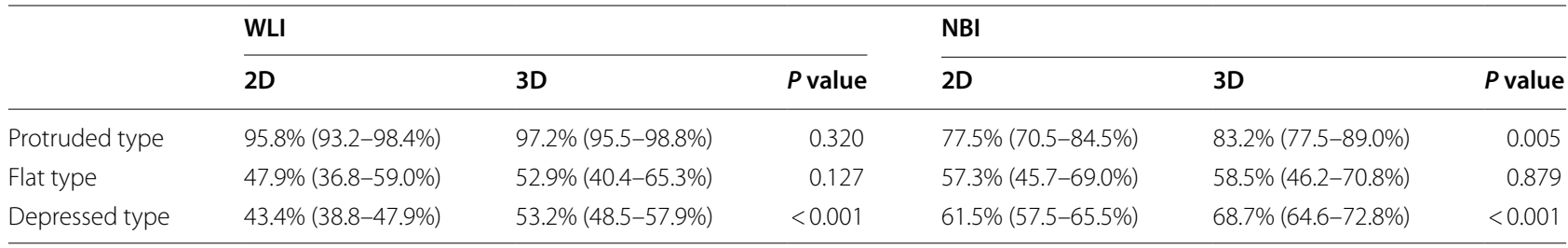

Data are presented as the mean ( $95 \%$ confidence interval)

WLI white-light imaging, NBI narrow-band imaging, 2D 2-dimensional, 3D 3-dimensional 
Table 4 The accuracy of the morphology type

\begin{tabular}{|c|c|c|c|c|c|c|}
\hline & \multicolumn{3}{|l|}{ WLI } & \multicolumn{3}{|l|}{ NBI NBI } \\
\hline & 2D & $3 \mathrm{D}$ & $P$ value & $2 \mathrm{D}$ & $3 D$ & $P$ value \\
\hline Overall & $66.0 \%(61.0-72.0 \%)$ & $71.0 \%(66.0-76.0 \%)$ & 0.104 & $69.0 \%(63.0-74.0 \%)$ & $76.0 \%(71.0-80.0 \%)$ & 0.004 \\
\hline Novice & $49.0 \%$ (39.0-59.0\%) & $60.0 \%(50.0-69.0 \%)$ & 0.043 & $52.0 \%(42.0-61.0 \%)$ & $67.0 \%(58.0-76.0 \%)$ & 0.005 \\
\hline Trainee & $76.0 \%$ (68.0-84.0\%) & $79.0 \%(71.0-87.0 \%)$ & 0.648 & $77.0 \%(69.0-85.0 \%)$ & $83.0 \%$ (76.0-90.0\%) & 0.118 \\
\hline Expert & $74.0 \%(65.0-83.0 \%)$ & $74.0 \%$ (65.0-83.0\%) & 1.0 & $77.0 \%$ (69.0-85.0\%) & $77.0 \%$ (69.0-85.0\%) & 1.0 \\
\hline
\end{tabular}

Data are presented as the mean (95\% confidence interval)

WLI white-light imaging, NBI narrow-band imaging, 2D 2-dimensional, 3D 3-dimensional

Table 5 The comparison of the ease of endoscopic lesion recognition

\begin{tabular}{|c|c|c|c|c|c|c|}
\hline & \multicolumn{2}{|l|}{ WLI } & \multirow[t]{2}{*}{$P$ value } & \multicolumn{2}{|l|}{ NBI } & \multirow[t]{2}{*}{$P$ value } \\
\hline & $2 \mathrm{D}$ & $3 D$ & & $2 \mathrm{D}$ & $3 D$ & \\
\hline Lesion morphology & $4.78(2.75)$ & $7.03(2.84)$ & $<0.001$ & $5.85(2.28)$ & $7.68(2.34)$ & $<0.001$ \\
\hline LESION extent & $4.18(2.75)$ & $6.05(2.86)$ & $<0.001$ & $5.07(2.49)$ & $6.76(2.53)$ & $<0.001$ \\
\hline $\begin{array}{l}\text { Comprehensive endoscopic } \\
\text { cognition }\end{array}$ & $4.28(2.81)$ & $6.21(2.98)$ & $<0.001$ & $5.35(2.42)$ & $7.18(2.54)$ & $<0.001$ \\
\hline
\end{tabular}

Data are presented as the mean (standard deviation)

WLI white-light imaging, NBI narrow-band imaging, 2D 2-dimensional, 3D 3-dimensional

delineation of the tumor margin and may thus enhance the curability of ESD for superficial gastrointestinal neoplasia.

Chromoendoscopy with indigo carmine has enabled the accurate diagnosis of the horizonal margin in $80 \%$ of EGCs $[17,18]$. It was particularly highly effective for protruded type 0 -IIa and 0 -I and depressed type 0 -IIC lesions, although successful delineation was achieved in only $32.1 \%$ of EGCs for flat type 0-IIb. Iizuka et al. reported that the addition of acetic acid spray before dispersing indigo carmine clarified the tumor margin compared with only indigo carmine [19]. Several studies found that magnifying endoscopy with NBI (M-NBI) was more useful than chromoendoscopy for delineating the lateral margin of EGC [20]. M-NBI successfully defined the lateral margin of $72.6 \%$ of EGCs with unsuccessful delineation on chromoendoscopy [17]. In contrast, a multicenter randomized study revealed that chromoendoscopy and M-NBI were clinically equivalent for determining the horizonal margin of EGC [21]. In the present study, the delineation of the tumor margin for superficial depressed type was more accurate under 3D than 2D visualization with both WLI and NBI. Three-dimensional visualization can identify subtle changes in the gastric mucosal epithelium by providing depth perception. In contrast, there was no significant difference between 2 and 3D visualization with WLI for superficial protruded type, although the accuracy of the lesion extent under 3D visualization was greater than that under $2 \mathrm{D}$ visualization with NBI, possibly due to the fact that the concordance rate of the lesion extent with WLI exceeded 95\% even with $2 \mathrm{D}$, meaning that recognizing the lateral margin in the protruded type is relatively easy.

There were only three flat-type EGCs, and while they were difficult to statistically evaluate, the $2 \mathrm{D}$ and $3 \mathrm{D}$ visualization results were equivalent. A previous study in which resected ESD specimens of superficial gastric tumor were evaluated also reported that $3 \mathrm{D}$ visualization enabled the accurate delineation of the lateral margin for type 0-IIc but not for type 0-IIb [22]. Due to the lack of any marked difference in the mucosal height from the surrounding mucosa in flat-type EGC, the accurate recognition of the tumor extent, even with various devices and techniques, is quite challenging. However, the accuracy of the lesion extent and the morphology type was higher with NBI than with WLI by $10 \%$ and $5 \%$ under 2D and 3D observation, respectively. Although the comparison of the still images of WLI and NBI was not impartial because both the WLI and NBI for each lesion were not same in this study, NBI may result in the better recognition of gastric lesions. Therefore, 3D-NBI observation is considered the most useful modality for detecting gastric cancerous lesions and recognizing the tumor extent at present.

The endoscopic diagnosis of EGC is usually affected by the experience and skill level of the endoscopist. Indeed, the accuracy of the tumor extent and morphology type in experts was quite high compared to that of the trainees 
under both $2 \mathrm{D}$ and $3 \mathrm{D}$ visualization in the present study. However, 3D visualization helped compensate for the lack of experience, allowing trainees to delineate margins as accurately as or more accurately than experts under 2D visualization. As mentioned in previous studies related to $3 \mathrm{D}$ visualization, one of the advantages of $3 \mathrm{D}$ endoscopy is its usefulness among endoscopists without sufficient experience in endoscopy [22, 23].

Several limitations associated with the present study warrant mention. First, the correct lesion margins constructed based on the mapping images in the pathological diagnosis may not be exactly the same as the actual lesion extent on endoscopic observation. Second, participants were not blinded to whether they were performing $2 \mathrm{D}$ or $3 \mathrm{D}$ visualization. Third, the number of subjects was relatively small, and this study was carried out at a single institution. Further studies to investigate the usefulness of 3D flexible endoscopy for the endoscopic diagnosis of gastrointestinal neoplasias are warranted.

\section{Conclusions}

In conclusion, 3D visualization improves the performance of the endoscopic diagnosis of superficial gastric neoplasias. These findings may improve the early detection of EGC and safe ESD without a positive margin for tumor cells.

\section{Abbreviations \\ ESD: Endoscopic submucosal dissection; EGC: Early gastric cancer; NBI: Narrow-band imaging; 3D: 3-Dimensional; 2D: 2-Dimensional; WLI: White- light imaging; VAS: Visual analog scaled; M-NBI: Magnifying endoscopy with narrow-band imaging.}

\section{Supplementary Information}

The online version contains supplementary material available at https://doi. org/10.1186/s12876-021-01829-y.

Additional file 1: Supplementary Table 1. The comparison of the coincidence rate of lesion extent in group A and group B. Supplementary Table 2. The comparison of the ease of endoscopic lesion recognition by the skill level of endoscopists.

\section{Acknowledgements}

We would like to thank Eigo Kodani, M.D., Masatoki Nakaza, M.D., Ryu Matsunaga, M.D. and Eriko Tominaga, M.D. at Nippon Medical School Hospital for participating in this study as novices.

\footnotetext{
Authors' contributions

$\mathrm{KH}$ and MK designed and conceived this study. KH, MK, HN, KK, EK, TU, TA, JO, $\mathrm{NA}, \mathrm{OG}, \mathrm{AT}$, and $\mathrm{Kl}$ collected the date. $\mathrm{KH}$ analyzed and interpreted the results and drafted of the manuscript. MK performed critical revision of the article for important intellectual content. All authors read and approved the final manuscript.
}

\section{Funding}

This research did not receive any funding.

\section{Availability of data and materials}

The datasets used and/or analyzed during the current study are available from the corresponding author on reasonable request.

\section{Declarations}

Ethics approval and consent to participate

This study was carried out in accordance with the Declaration of Helsinki and the ethical guidelines for medical and health research involving human subjects in Japan. This trial protocol was approved by the ethics committee at Nippon Medical School Hospital (30-05-923), and this study was registered at the University Hospital Medical Information (UMIN000033991). Written informed consent was obtained from all patients before the procedures.

\section{Consent for publication}

Not applicable.

\section{Competing interests}

The authors declare no conflicts of interest associated with this manuscript. The 3D endoscopy system used in this study was provided by Olympus Medical Systems Corp.

\section{Author details}

${ }^{1}$ Department of Gastroenterology, Nippon Medical School, Graduate School of Medicine, 1-1-5, Sendagi, Bunkyo-ku, Tokyo 113-8603, Japan. ${ }^{2}$ Department of Analytic Human Pathology, Nippon Medical School, Graduate School of Medicine, Tokyo, Japan.

Received: 23 January 2021 Accepted: 17 May 2021

Published online: 28 May 2021

\section{References}

1. Bray F, Ferlay J, Soerjomataram I, Siegel RL, Torre LA, Jemal A. Global cancer statistics 2018: GLOBOCAN estimates of incidence and mortality worldwide for 36 cancers in 185 countries. CA Cancer J Clin. 2018;68:394-424

2. Ono H, Kondo H, Gotoda T, Shirao K, Yamaguchi H, Saito D, et al. Endoscopic mucosal resection for treatment of early gastric cancer. Gut. 2001;48:225-9.

3. Watanabe K, Ogata S, Kawazoe S, Watanabe K, Koyama T, Kajiwara T, et al. Clinical outcomes of EMR for gastric tumors: historical pilot evaluation between endoscopic submucosal dissection and conventional mucosal resection. Gastrointest Endosc. 2006;63:776-82.

4. Pimentel-Nunes P, Libânio D, Lage J, Abrantes D, Coimbra M, Esposito G, et al. A multicenter prospective study of the real-time use of narrow-band imaging in the diagnosis of premalignant gastric conditions and lesions. Endoscopy. 2016;48:723-30

5. Kato M, Kaise M, Yonezawa J, Toyoizumi H, Yoshimura N, Yoshida Y, et al. Magnifying endoscopy with narrow-band imaging achieves superior accuracy in the differential diagnosis of superficial gastric lesions identified with white-light endoscopy: a prospective study. Gastrointest Endosc. 2010;72:523-9.

6. Dohi O, Yagi N, Majima A, Horii Y, Kitaichi T, Onozawa Y, et al. Diagnostic ability of magnifying endoscopy with blue laser imaging for early gastric cancer: a prospective study. Gastric Cancer. 2017;20:297-303.

7. Kitagawa Y, Suzuki T, Nankinzan R, Ishigaki A, Furukawa K, Sugita O, et al. Comparison of endoscopic visibility and miss rate for early gastric cancers after Helicobacter pylori eradication with white-light imaging versus linked color imaging. Dig Endosc. 2020;32:769-77.

8. Fergo C, Burcharth J, Pommergaard HC, Kildebro N, Rosenberg J. Threedimensional laparoscopy vs 2-dimensional laparoscopy with high-definition technology for abdominal surgery: a systematic review. Am J Surg. 2017;213:159-70.

9. Sørensen SM, Savran MM, Konge L, Bjerrum F. Three-dimensional versus two-dimensional vision in laparoscopy: a systematic review. Surg Endosc. 2016;30:11-23. 
10. Aradaib M, Neary P, Hafeez A, Kalbassi R, Parvaiz A, O'Riordain D. Safe adoption of robotic colorectal surgery using structured training: early Irish experience. J Robot Surg. 2019;13:657-62.

11. Nomura K, Kikuchi D, Kiase M, lizuka T, Ochiai Y, Suzuki Y, et al. Comparison of 3D endoscopy and conventional 2D endoscopy in gastric endoscopic submucosal dissection: an ex vivo animal study. Surg Endosc. 2019;33:4164-70.

12. Kikuchi D, Kaise M, Nomura K, Toba T, Kuribayashi Y, Tanaka M, et al. Feasibility study of the three-dimensional flexible endoscope in endoscopic submucosal dissection: an ex vivo animal study. Digestion. 2017:95:237-41.

13. Higuchi K, Kaise M, Noda H, Ikeda G, Akimoto T, Yamawaki H, et al. Usefulness of 3-dimensional flexible endoscopy in esophageal endoscopic submucosal dissection in an ex vivo animal model. Gastroenterol Res Pract. 2019;2019:4051956.

14. Chiu PWY, Yip HC, Xia XF, Chan SM, Ng EKW, Lau JYW. How I do it: flexible 3-D endoscope for endoscopic submucosal dissection. Dig Endosc. 2019;31:323-8.

15. Nomura K, Kikuchi D, Kaise M, lizuka T, Ochiai Y, Suzuki Y, et al. Operational effectiveness of three-dimensional flexible endoscopy: an ex vivo study using a new model. Surg Endosc. 2019;33:3612-5.

16. Japanese Gastric Cancer Association. Japanese classification of gastric carcinoma: 3rd English edition. Gastric Cancer. 2011;14:101-12.

17. Nagahama T, Yao K, Maki S, Yasaka M, Takaki Y, Matsui T, et al. Usefulness of magnifying endoscopy with narrow-band imaging for determining the horizontal extent of early gastric cancer when there is an unclear margin by chromoendoscopy. Gastrointest Endosc. 2011;74:1259-67.

18. Yoshinaga S, Gotoda T, Oda I, Saito Y, Matsuda T, Nakajima T, et al. Clinical imaging of early gastric cancers-conventional endoscopy: including chromoendoscopy using indigo carmine. Stomach Intestine. 2009:44:650-62.

19. lizuka T, Kikuchi D, Hoteya S, Yahagi N. The acetic acid + indigocarmine method in the delineation of gastric cencer. J Gastroenterol Hepatol. 2008;23:1358-61.

20. Kiyotoki S, Nishikawa J, Satake M, Fukagawa Y, Shirai Y, Hamabe K, et al. Usefulness of magnifying endoscopy with narrow-band imaging for determining gastric tumor margin. J Gastroenterol Hepatol. 2010;25:1636-41.

21. Nagahama T, Yao K, Uedo N, Doyama H, Ueo T, Uchita K, et al. Delineation of the extent of early gastric cancer by magnifying narrow-band imaging and chromoendoscopy: a multicenter randomized controlled trial. Endoscopy. 2018;50:566-76.

22. Nomura K, Kaise M, Kikuchi D, lizuka T, Fukuma Y, Kuribayashi Y, et al. Recognition accuracy of tumor extent using a prototype 3D endoscope for superficial gastric tumor: an ex vivo crossover study. Endosc Int Open. 2018;6:E652-8.

23. Nomura K, Kaise M, Kikuchi D, lizuka T, Fukuma Y, Kuribayashi Y, et al. Recognition accuracy using 3D endoscopic images for superficial gastrointestinal cancer: a crossover study. Gastroenterol Res Pract. 2016;2016:4561468

\section{Publisher's Note}

Springer Nature remains neutral with regard to jurisdictional claims in published maps and institutional affiliations.
Ready to submit your research? Choose BMC and benefit from:

- fast, convenient online submission

- thorough peer review by experienced researchers in your field

- rapid publication on acceptance

- support for research data, including large and complex data types

- gold Open Access which fosters wider collaboration and increased citations

- maximum visibility for your research: over 100M website views per year

At BMC, research is always in progress.

Learn more biomedcentral.com/submissions 